\title{
Factores de riesgo para preeclampsia en un hospital de la amazonia peruana
}

\author{
Sally Torres-Ruiz
}

RESUMEN: Objetivo: Determinar la asociación entre los factores riesgo y la presencia de preeclampsia. Metodología: estudio retrospectivo de casos y controles mediante la recopilación de historias clínicas de gestantes atendidas en el Hospital Iquitos César Garayar García de Iquitos-Perú de enero a septiembre del 2015. Para el grupo de los casos (gestantes con preeclampsia) la muestra fue de 80 mujeres. Para el grupo de controles (gestantes sin diagnóstico de preeclampsia) la muestra fue de 80. Para el análisis bivariado se aplicó la prueba de chi cuadrado. Asimismo se empleó un análisis multivariado con regresión logística para calcular Odds Ratio (OR) con intervalos de confianza al 95\%. Resultados: los factores de riesgo asociados a la preeclampsia fueron: edad mayor de 35 años (OR:1.6); residir en zona rural (OR:2.2); educación primaria o sin estudios (OR:1.6); nuliparidad (OR:1.2); edad gestacional de 32-36 semanas (OR:2.9); tener entre cero y cinco controles prenatales (OR:6.3); tener antecedentes familiares (OR:10.6) y personales (OR:40.1) de preeclampsia. Conclusiones: los factores riesgo para preeclampsia en la región selva son: tener de 35 años a más, residir en zona rural, poseer un grado de instrucción primaria o sin estudios, ser nulípara y haber realizado solamente de cero a cinco controles prenatales. La residencia en zona rural constituye un factor de riesgo importante en la preeclampsia en la región amazónica. Las gestantes de estas zonas están expuestas a una menor participación a los controles prenatales debido a un desconocimiento asociado a niveles de instrucción bajos que puede generar temor antes los controles.

PALABRAS CLAVES:

Citar como:
Preeclampsia; Factores de riesgo; Factores asociados.

Torres S. Factores de riesgo para preeclampsia en un hospital de la amazonia peruana. CASUS. 2016; 1(1): 18-26. 


\section{INTRODUCCIÓN}

La preeclampsia es una patología de múltiples causas que afecta a las gestantes. Se desconoce su origen y su tratamiento definitivo es culminar el embarazo, a través del parto. Es motivo de secuelas en la madre y en el neonato (1), trae como consecuencia hospitalizaciones recurrentes, perjudicando económicamente a la familia, centros de salud y en general al sistema de salud de cada país. La preeclampsia se caracteriza por la presencia de la hipertensión y proteinuria después de la semana 20 del embarazo, la misma puede acompañarse de edemas (1).

A nivel mundial, el $75 \%$ de la mortalidad materna se asocia a las siguientes complicaciones: las hemorragias graves, las infecciones y la hipertensión gestacional (preeclampsia y eclampsia) (2). Entre las principales causas de muerte materna en Perú se encuentran: las hemorragias obstétricas, los trastornos hipertensivos en el embarazo, parto y puerperio y las complicaciones no obstétricas (3). Por otra parte, entre las principales causas de muerte materna en la región Loreto se reportan: las hemorragias, los trastornos hipertensivos en el embarazo y las infecciones obstétricas. Las mismas se evidencian en el Hospital Iquitos César Garayar García (3).

La mayoría de los casos de preeclampsia ocurren en gestantes saludables. Por ello es importante establecer los factores de riesgo que pueden influir en el desarrollo de esta patología. Entre los cuales cabe mencionar: edad materna extrema (4-6), vivir en zona rural $(3,7)$, grado de instrucción primaria $(8,9)$, paridad (nuliparidad) $(5,7,10)$, mayor edad gestacional $(7,10)$ un control prenatal inadecuado. Así como presentar comorbilidad, entre ellas: hipertensión arterial (11), obesidad $(11,12)$, diabetes (11) y haber presentado antecedentes personales (13-15) y familiares de preeclampsia $(16,17)$. Sin embargo, existen dificultades para la identificación previa a las manifestaciones clínicas. Una consideración desde el punto de vista epidemiológico permite identificar en la población femenina factores de riesgo asociados al desarrollo de la patología. Considerando que la misma afecta a un gran número de gestantes en la ciudad de Iquitos (región selva del Perú), su relevancia como problema de salud prenatal y social se realiza el presente estudio. El objetivo propuesto fue determinar la asociación entre los factores de riesgo (sociodemográficos y obstétricos) y la presencia de preeclampsia en gestantes atendidas en el Hospital Iquitos César Garayar García durante el periodo de enero a septiembre del 2015.

\section{MATERIALES Y MÉTODOS}

Estudio retrospectivo de casos y controles mediante la recopilación de historias clínicas. Para determinar los grupos de casos y controles se consideró una población de 3284 gestantes atendidas en el Hospital entre los meses de enero a septiembre del 2015. Para el grupo de los casos (gestantes con preeclampsia) se tuvo en consideración a 150 gestantes. A partir del uso de la fórmula del tamaño muestral se seleccionaron 108 mujeres. Se excluyeron 28 gestantes por presentar datos incompletos en la historia clínica. Para el grupo de controles se obtuvo una muestra de 120 mujeres sin el diagnóstico de preeclampsia, sin embargo la muestra final fue de 80 gestantes debido a que 40 de ellas tenían datos incompletos en la historia clínica. Se obtuvo una muestra efectiva de 160 gestantes. Ver figura 1.

\section{Figura 1. Diagrama de flujo para determinar el tamaño muestral}

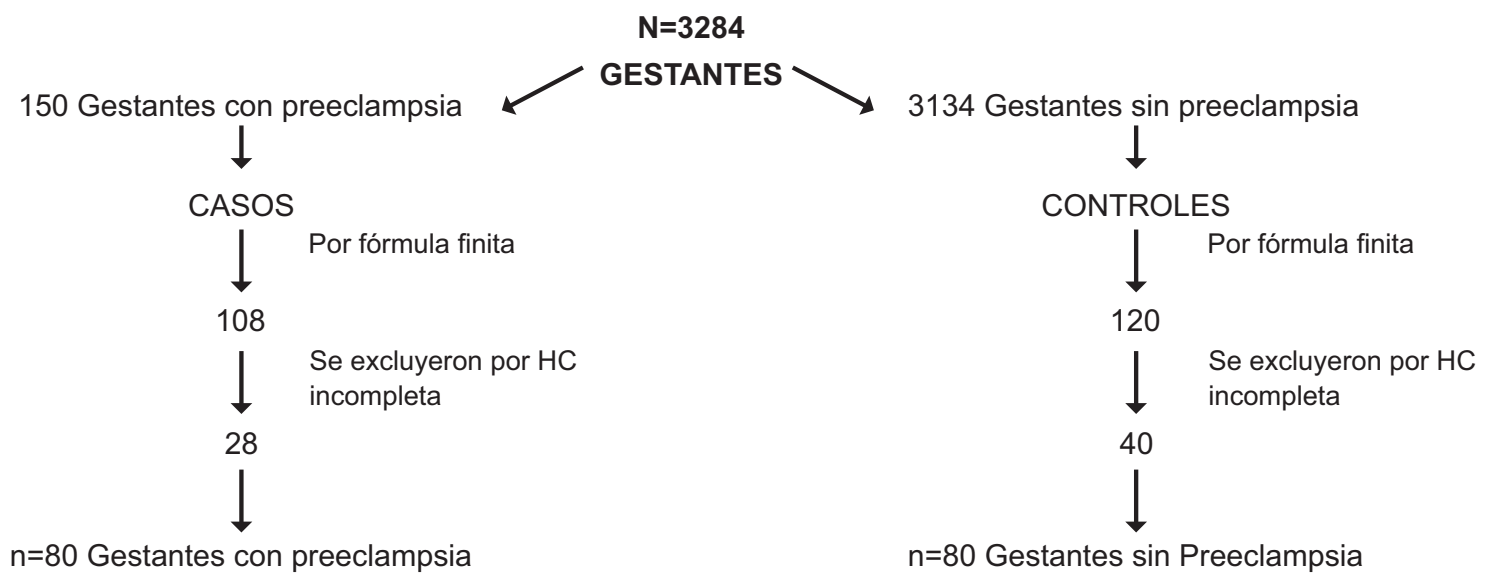


Para la recolección de datos se revisó en primera instancia el libro de Registro de Información de Hospitalización del Servicio de Maternidad/Centro Obstétrico y el Sistema Informático Estadístico Perinatal (SIP 2000) con el fin de obtener los números de las historias clínicas de las gestantes con preeclampsia. Por último, a través de las historias clínicas se identificaron los datos personales y los antecedentes obstétricos de las gestantes que presentaron el diagnóstico de preeclampsia leve o severa.

La información de los casos y controles fue registrada en una ficha de recolección de datos. Con la misma se registraron los siguientes datos sociodemográficos: edad (variable categorizada en: $<20$ años, 20 a 34 años, 35 años a más); lugar de residencia: urbana (que procede de zona dentro del perímetro urbano de la ciudad), urbano-marginal (que procede de zona alejada del perímetro urbano) y rural (que procede de pueblos o caseríos aledaños a la ciudad). Nivel de instrucción (sin estudios, primaria, secundaria, técnica y universitaria). En la segunda sección de la ficha de datos se registraron los antecedentes obstétricos. Paridad: nulípara (0 partos), uno a más hijos; período intergenésico (no registrado, $<2$ años, 2 años a más); edad gestacional ( $\leq 31$ semanas, entre 32 a 36 semanas $\mathrm{y} \geq 37$ semanas); número de controles prenatales ( 0 a 5 , 6 a más); comorbilidad (sí, no); antecedentes familiares de preeclampsia (sí, no) y antecedentes personales de preeclampsia (sí, no).
Se aplicó la prueba de chi cuadrado para variables categóricas. Asimismo se empleó un análisis multivariado con regresión logística para calcular Odds Ratio (OR) con intervalos de confianza al $95 \%$. Se consideró una significancia estadística menor a 0.05 para medir la magnitud de riesgo entre las variables. Para el análisis estadístico se utilizó el programa SPSS versión 21.

El presente estudio fue revisado y aprobado por el Comité de Ética de Investigación del Hospital Iquitos César Garayar García y de la Universidad Nacional de la Amazonía Peruana. La realización del mismo tuvo en cuenta la protección de los datos de los sujetos involucrados.

\section{RESULTADOS}

De las 3284 gestantes que se registraron en el Hospital la prevalencia de preeclampsia fue de $2,4 \%$. En cuanto al tipo de preeclampsia la más frecuente fue la tipo leve (58\%) y la severa se presentó en un $42 \%$. En la Tabla 1 se muestra que todos los factores de riesgo resultaron estadísticamente significativos para presentar preeclampsia. Entre los valores que destacaron fueron: la edad de las gestantes menor a 20 años $(46 \%)$; residir en zona rural (47\%) y un nivel de instrucción secundario $(60 \%)$.

Tabla 1. Características sociodemográficas de las gestantes atendidas en el Hospital lquitos

\begin{tabular}{|c|c|c|}
\hline Factores de riesgo & Preeclampsia n(\%) & No preeclampsia $n(\%)$ \\
\hline \multicolumn{3}{|l|}{ Edad (años) } \\
\hline 20 años & $37(46)$ & $20(25)$ \\
\hline 20 a 34 años & $13(16)$ & $50(62)$ \\
\hline 35 años a más & $30(37)$ & $10(12)$ \\
\hline \multicolumn{3}{|l|}{ Procedencia } \\
\hline Urbano & $14(17)$ & $20(25)$ \\
\hline Urbano-Marginal & $28(35)$ & $36(45)$ \\
\hline Rural & $38(47)$ & $24(30)$ \\
\hline \multicolumn{3}{|l|}{ Nivel de instrucción } \\
\hline Sin estudios & $0(0)$ & $2(2)$ \\
\hline Primaria & $22(27)$ & $18(22)$ \\
\hline Secundaria & 48(60) & $49(61)$ \\
\hline Técnico & $7(8)$ & $7(8)$ \\
\hline Superior & $3(3)$ & $4(5)$ \\
\hline
\end{tabular}

Nota: El estadístico empleado fue el chi cuadrado. Todas las variables reportaron significancia estadística $(p<0.05)$. 
Respecto a los factores de riesgo obstétricos para presentar preeclampsia todos resultaron estadísticamente significativos excepto el periodo intergenésico. Destacaron los siguientes valores: nuliparidad (33\%); edad gestacional $\geq 37$ semanas
(57\%), tener de cero a cinco controles prenatales $(77 \%)$, antecedentes personales (64\%) y familiares $(52 \%)$ de preeclampsia. Estos resultados se pueden ver en la Tabla 2.

Tabla 2. Características obstétricas de las gestantes atendidas

\begin{tabular}{|c|c|c|}
\hline Factores de riesgo & Preeclampsia n(\%) & No preeclampsia $n(\%)$ \\
\hline \multicolumn{3}{|l|}{ Paridad } \\
\hline Nuliparidad & $27(33)$ & $31(38)$ \\
\hline Primípara & $17(21)$ & 28(35) \\
\hline Secundípara & 18(22) & $15(18)$ \\
\hline Multípara & $12(15)$ & $3(3)$ \\
\hline Gran multípara & $6(7)$ & $3(3)$ \\
\hline \multicolumn{3}{|l|}{ Periodo intergenésico* } \\
\hline No registrado & $27(34)$ & $31(38)$ \\
\hline$<2$ años & $4(5)$ & 16(20) \\
\hline 2 años a más & $49(61)$ & $33(42)$ \\
\hline \multicolumn{3}{|l|}{ Edad gestacional } \\
\hline$\leq 31$ semanas & $2(2)$ & $2(2)$ \\
\hline 32 a 36 semanas & $32(40)$ & $15(18)$ \\
\hline$\geq 37$ semanas & $46(57)$ & $63(78$ \\
\hline \multicolumn{3}{|c|}{ Número de controles prenatales } \\
\hline $0-5$ controles & $62(77)$ & $28(35)$ \\
\hline 6 a más controles & $18(22)$ & $52(65$ \\
\hline \multicolumn{3}{|l|}{ Comorbilidad } \\
\hline Sí & $72(90)$ & $29(36)$ \\
\hline No & $8(10)$ & $51(64)$ \\
\hline \multicolumn{3}{|l|}{ Antecedentes familiares } \\
\hline Sí & $29(36)$ & $4(5)$ \\
\hline No & $51(64)$ & 76 (95) \\
\hline \multicolumn{3}{|l|}{ Antecedentes personales } \\
\hline Sí & $41(52)$ & $2(3)$ \\
\hline No & $39(48)$ & 78(97) \\
\hline
\end{tabular}

Nota: El estadístico empleado: chi cuadrado. Todas las variables mostraron valores significativos $p<0.05$ *No mostró significancia 
En la Tabla 3 se muestra el análisis del modelo de regresión logística entre los factores sociodemográficos y la presencia de preeclampsia. Las gestantes con más de 34 años tuvieron 0.6 veces más probabilidades de tener preeclampsia en comparación con las que tenían menos de 20 años (OR: 1.6; IC 95\% 0.67-4.10). Las que residían en zona rural tuvieron 1.2 veces más probabilidades de tener preeclampsia que las que residían en zona urbana (OR: 2.2; IC 95\% 0.95 5.37). La educación incide positivamente en el riesgo de tener preeclampsia. Las gestantes con educación primaria o sin estudios tuvieron 0.6 más probabilidades de tener preeclampsia en comparación con las que cuentan con educación superior (OR: 1.6; IC $95 \%$ 0.29-9.6).

\section{Tabla 3. Asociación entre los factores sociodemográficos y preeclampsia en las gestantes}

\begin{tabular}{lccc}
\hline \multicolumn{1}{c}{ Factores de riesgo } & OR & IC & $p$ \\
\hline Edad (años) & & & - \\
$<20$ años & 1.0 & - & $<0.001$ \\
20 a 34 años & 0.1 & $0.06-0.32$ & $<0.001$ \\
35 años a más & 1.6 & $0.67-4.10$ & \\
& & & - \\
Procedencia & & - & $<0.001$ \\
Urbano & 1.0 & $0.47-2.62$ & \\
Urbano-marginal & 1.1 & $0.95-5.37$ & - \\
Rural & 2.2 & & $<0.001$ \\
& & - & $<0.001$ \\
Nivel de instrucción & 1.0 & $0.27-11.3$ & $<0.001$ \\
Superior & 1.6 & $0.26-7.3$ & \\
Técnico & 1.3 & $0.29-9.6$ & \\
Secundaria & 1.6 & & \\
Primaria/sin estudios & & & \\
\hline
\end{tabular}

Respecto al modelo de regresión logística entre los factores obstétricos y la presencia de preeclampsia las gestantes nulíparas tienen 0.2 veces más probabilidades de tener preeclampsia en comparación con las que tuvieron de uno a más hijos (OR: 1.2; IC $95 \% 0.60-2.27)$. La posibilidad de tener preeclampsia fue 1.9 más veces para las gestantes que tuvieron una edad gestacional entre 32 a 36 semanas en comparación con las que tenían de 37 a más semanas (OR: 2.9; IC 95\% 1.42-6.10). La cantidad de controles prenatales incide positivamente en las probabilidades de tener preeclampsia. Haber tenido entre cero y cinco controles prenatales aumenta de 5.3 la posibilidad de tener preeclampsia respecto que las que presentaron seis o más controles prenatales (OR: 6.3; IC 95\% 3.1712.9). La comorbilidad tiene un importante efecto en la preeclampsia. Las gestantes con comorbilidad tuvieron 14.5 más veces de tener preeclampsia en comparación con las que no tuvieron (OR: 15.5; IC $95 \%$ 6.75-38.9). Tener un antecedente familiar o personal incide positivamente en la preeclampsia. Las gestantes con antecedentes familiares tuvieron 9.6 veces más posibilidades de presentar preeclampsia en comparación con las que no tuvieron antecedentes familiares (OR: 10.6; IC 95\% 3.76-37.4). Ver Tabla 4. 
Tabla 4. Asociación entre los factores obstétricos y preeclampsia en las gestantes

\begin{tabular}{|c|c|c|c|}
\hline Factores de riesgo & OR & IC & $p$ \\
\hline \multicolumn{4}{|l|}{ Paridad } \\
\hline De uno a más & 1.0 & - & - \\
\hline Nulípara & 1.2 & $0.60-2.27$ & $<0.001$ \\
\hline \multicolumn{4}{|l|}{ Edad gestacional } \\
\hline$\leq 37$ semanas & 1.0 & - & - \\
\hline 32 a 36 semanas & 2.9 & $1.42-6.10$ & $<0.001$ \\
\hline$\geq 31$ semanas & 1.4 & $0.14-13.5$ & $<0.001$ \\
\hline \multicolumn{4}{|c|}{ Número de controles prenatales } \\
\hline 6 a más controles & 1.0 & - & - \\
\hline $0-5$ controles & 6.3 & $3.17-12.9$ & $<0.001$ \\
\hline \multicolumn{4}{|l|}{ Comorbilidad } \\
\hline No & 1.0 & - & - \\
\hline Sí & 15.5 & $6.75-38.9$ & $<0.001$ \\
\hline \multicolumn{4}{|l|}{ Antecedentes familiares } \\
\hline No & 1.0 & - & - \\
\hline Sí & 10.6 & $3.76-37.4$ & $<0.001$ \\
\hline \multicolumn{4}{|l|}{ Antecedentes personales } \\
\hline No & 1.0 & - & - \\
\hline Sí & 40.1 & $10.7-25.7$ & $<0.001$ \\
\hline
\end{tabular}

\section{DISCUSIÓN}

Entre los factores de riesgo más importantes para presentar preeclampsia fueron: tener 35 años o más; tener residencia rural y un nivel de instrucción primaria o sin estudios. Respecto a los factores obstétricos fueron: nuliparidad, edad gestacional de 32-36 semanas; controles prenatales insuficientes y la presencia de antecedentes personales y familiares de preeclampsia.

La edad mayor o igual a 35 años de la gestante fue un factor de riesgo para preeclampsia. Estudios similares reportaron que las gestantes que tenían más de 34 años presentan más riesgo de presentar preeclampsia $(4,5)$. Esto podría deberse a que las mujeres gestantes mayores de 35 años tienen más probabilidades de presentar hipertensión arterial (6). La edad entre 20 y 34 años resulta un factor protector para preeclampsia. Lo cual es confirmado por la literatura que indica una mayor predisposición en adolescentes y en mujeres mayores de 34 años $(18,19)$.
Referente a la residencia en zona rural esta fue un factor de riesgo de preeclampsia. En otro estudio en la misma región también se encontró asociación significativa entre ambas variables (7). Esta asociación pudiera deberse a que las gestantes provenientes de zonas rurales posiblemente experimenten temor ante los controles prenatales debido a un desconocimiento asociado a niveles de instrucción bajos, conjuntamente al aumento de las distancias a recorrer hacia el centro de salud más cercano, lo que supone a su vez un incremento de los costos en la atención prenatal (3).

Respecto al factor grado de instrucción se encontró asociación significativa entre el nivel de instrucción primaria y la presencia de preeclampsia. Un estudio propone hallazgos similares en gestantes con dicha patología (8). Esto debido a que las analfabetas y con escasos recursos son las más susceptibles a desarrollar esta patología por la falta de conocimientos relativos a la prevención de la misma (9). En cuanto al grado de 
instrucción técnico, presentan más riesgo de desarrollar la patología que las gestantes que tienen grado de instrucción secundaria. Esto pudiera deberse a que con un aumento del grado de instrucción generalmente se posterga el inicio de la maternidad. Este factor, de igual manera, se encuentra relacionado con la nuliparidad $(5,7)$ y con el aumento de la edad al nacimiento del primer hijo (20).

Respecto a la cantidad de controles prenatales (cero a cinco) y preeclampsia se encontró asociación significativa información que es confirmada por otros estudios $(8,11)$. Esto pudiera deberse a que con una cantidad insuficiente de controles prenatales no será posible un seguimiento oportuno del embarazo, ni detectar sus comorbilidades y complicaciones. De esta manera no es consentido mejorar el estilo de vida de la paciente (4).

Respecto a los antecedentes personales y la presencia de preeclampsia, de acuerdo con estudios similares, se encontró asociación significativa entre ambos factores $(13,14)$. Esta podría ser originada porque existe una predisposición a la enfermedad, siendo importante en la manifestación de la misma el factor genético, ya que utiliza como mediador al sistema inmunológico (15). En relación a los antecedentes familiares estudios similares confirman esta asociación $(10,16)$. Existe una hipótesis acerca de dicha relación del antecedente personal que es debido que a la transmisión recesiva de genes maternos (17).

Entre las principales limitaciones del estudio se mencionan: el sesgo durante la selección. Se tuvo que excluir a gestantes tanto en el grupo de los casos como en los controles por presentar datos incompletos en las historias clínica y porque mostraron una discordancia entre la definición de preeclampsia y los datos reflejados. Entre las fortalezas del estudio destacan el tipo de diseño retrospectivo de casos y controles, el análisis estadístico empleado, la muestra representativa de la región amazónica y el presentar información epidemiológica regional de factores de riesgo de la enfermedad.

\section{CONCLUSIONES}

Se evidencia que los factores riesgo para preeclampsia en la población peruana de la región selva son: tener de 35 años a más, residir en zona rural, poseer un grado de instrucción primaria o sin estudios, ser nulípara, tener una edad gestacional entre 32 y 36 semanas y haber realizado solamente de cero a cinco controles prenatales.

La residencia en zona rural constituye un factor de riesgo importante en la preeclampsia en la región amazónica. Las gestantes de estas zonas están expuestas a una menor participación a los controles prenatales debido a un desconocimiento asociado a niveles de instrucción bajos que puede generar temor antes los controles. Por otro lado, las mayores distancias a recorrer hacia los centros de salud comportan un aumento de los costos de la atención al embarazo. Una cantidad insuficiente de controles prenatales no permite un oportuno seguimiento del embarazo, ni la mejora de los estilos de vida de las gestantes.

La preeclampsia es un problema de salud del área de Ginecología y Obstetricia, por ello se recomienda seguir haciendo estudios de investigación sobre esta patología, ya que cuenta con una gran incidencia a nivel nacional y en la zona de la selva peruana (Loreto) ocasionando secuelas desfavorables tanto para la madre como para los recién nacidos. Estos estudios contribuirían al desempeño eficaz en cuanto a prevención y promoción de salud de las gestantes. Se sugiere también promover campañas de concientización a la población, dirigidas específicamente, a las mujeres en edad reproductiva y las que tienen algún factor de riesgo para preeclampsia con la finalidad de incentivarlas a la realización de un adecuado control prenatal y así evitar complicaciones durante el embarazo y el parto. También se recomienda que el Sistema de Salud mejore la calidad del control prenatal, a través de la capacitación del personal de salud para un mejor desempeño en la prevención y promoción de la salud en gestantes con riesgo que se encuentran en las zonas rurales, por tener menos acceso a los controles prenatales.

\section{AGRADECIMIENTOS}

A las autoridades y el personal de estadística del Hospital Iquitos CGG por las facilidades brindadas en la recolección de datos. 


\section{REFERENCIAS BIBLIOGRÁFICAS}

1. Sibai B, Dekker G, Kupferminc M. Pre-eclampsia. The Lancet. 2005;365(9461):785-799.

2. Say L, Chou D, Gemmill A, Tunçalp Ö, Moller AB, Daniels JD, et al. Global Causes of Maternal Death: A WHO Systematic Analysis. Lancet Global Health. 2014;2(6):323-333.

3. Ministerio de Salud de Perú. Salud materna [Internet]. Lima, Perú: INEI; 2011 (citado 05 de octubre de 2016). Disponible en:

http://www.minsa.gob.pe/portalweb/ 06 prevencion/prevencion_7.asp?sub $5: 7$

4. Benites $\mathrm{Y}$, Bazán S, Valladares D. Factores asociados al desarrollo de preeclampsia en un Hospital de Piura, Perú. CIMEL. 2011;16(2):77-82

5. Heredia IC. Factores de riesgo asociados a Preeclampsia en el Hospital Regional de Loreto de enero 2010 a diciembre 2014 [Tesis]. Universidad Privada Antenor Orrego; 2015.

6. Bianco A, Stone J, Lynch L, Lapinski R, Berkowitz G, Berkowitz R.Pregnancy outcome at Age 40 and Older. 1996;87(6):917-922.

7. Flores M. Factores de riesgo materno de preeclampsia en mujeres en edad fértil atendidas en el Hospital Regional de Loreto durante el año 2014. [Tesis]. Universidad nacional de laAmazonia Peruana; 2014.
8. Bosmediano N. Factores de riesgo en pacientes gestantes añosas con preeclampsia en Hospital III IquitosEsSalud 2013 [Tesis]. Universidad Nacional de la Amazonia Peruana; 2014

9. Amarán JE, Sosa M, Pérez M, Arias Acosta D, Valverde Bravo I. Principales características de la preeclampsia grave en gestantes ingresadas en un hospital de Zimbabwe. MEDISAN. 2009;13(3).

10. Cruz J, Hernández P, Yanes M, Isla A. Factores de riesgo de preeclampsia: enfoque inmunoendocrino. Parte I. Revista Cubana de Medicina General Integral. 2007;23(4).

11. Díaz J. Factores de riesgo para preeclampsia en pacientes adolescentes atendidas en el Hospital Nacional Sergio E. Bernales en el año 2015 [Tesis]. Universidad Ricardo Palma; 2016.

12. Teppa AD, Terán J. Factores de riesgo asociados con la preeclampsia. Rev Obstet Ginecol Venez. 2001;61(1):4956.

13. Morgan F, Calderón S, Martínez J, González A, Quevedo E. Factores de riesgo asociado con preeclampsia: estudio de casos y controles. Ginecol Obstet Mex. 2010;78(3):153-159.

14. Morales Ruiz C. Factores de riesgo asociados a preeclampsia en el Hospital Nacional Daniel Alcides Carrión. Callao. Abril a junio de 2010.
Rev. Perú Epidemiología. 2011;15(2):97-101.

15. Saftlas AF, Olson DR, Franks AL, Atrash HK, Pokras R. Epidemiology of preeclampsia and eclampsia in the United States, 1979-1986. American journal of obstetrics and gynecology, 1990; 163(2):460-465.

16. Valdés M, Hernández J. Factores de riesgo para preeclampsia. Revista Cubana de Medicina Militar. 2014;43(3):307-316.

17. Liston WA, Kilpatrick DC. Is genetic susceptibility to preeclampsia conferred by homozygosity for the same single recessive gene in mother and fetus? BJOG: An International Journal of Obstetrics and Gynaecology. 2011;98(11):1079-86.

18. Lamminpää R, Vehviläinen-Julkunen $\mathrm{K}, \mathrm{G}$ issler $\mathrm{M}$, Heinonen $\mathrm{S}$. Preeclampsia complicated by advanced maternal age: a registrybased study on primiparous women in Finland 1997-2008. BMC pregnancy and childbirth. 2012;12(47):1-5.

19. Pacheco J, Villacorta A, Del Carpio L, Velásquez É, Acosta O. Repercusión de la preeclampsia/ eclampsia en la mujer peruana y su perinato, 20002006. Revista Peruana de Ginecología y Obstetricia. 2014;60(4):279-290.

20. Mills M, Rindfuss RR, McDonald P, Te Velde E. Why do people postpone parenthood? Reasons and social policy incentives. 


\section{Risk factors for preeclampsia in a hospital in the Peruvian Amazon}

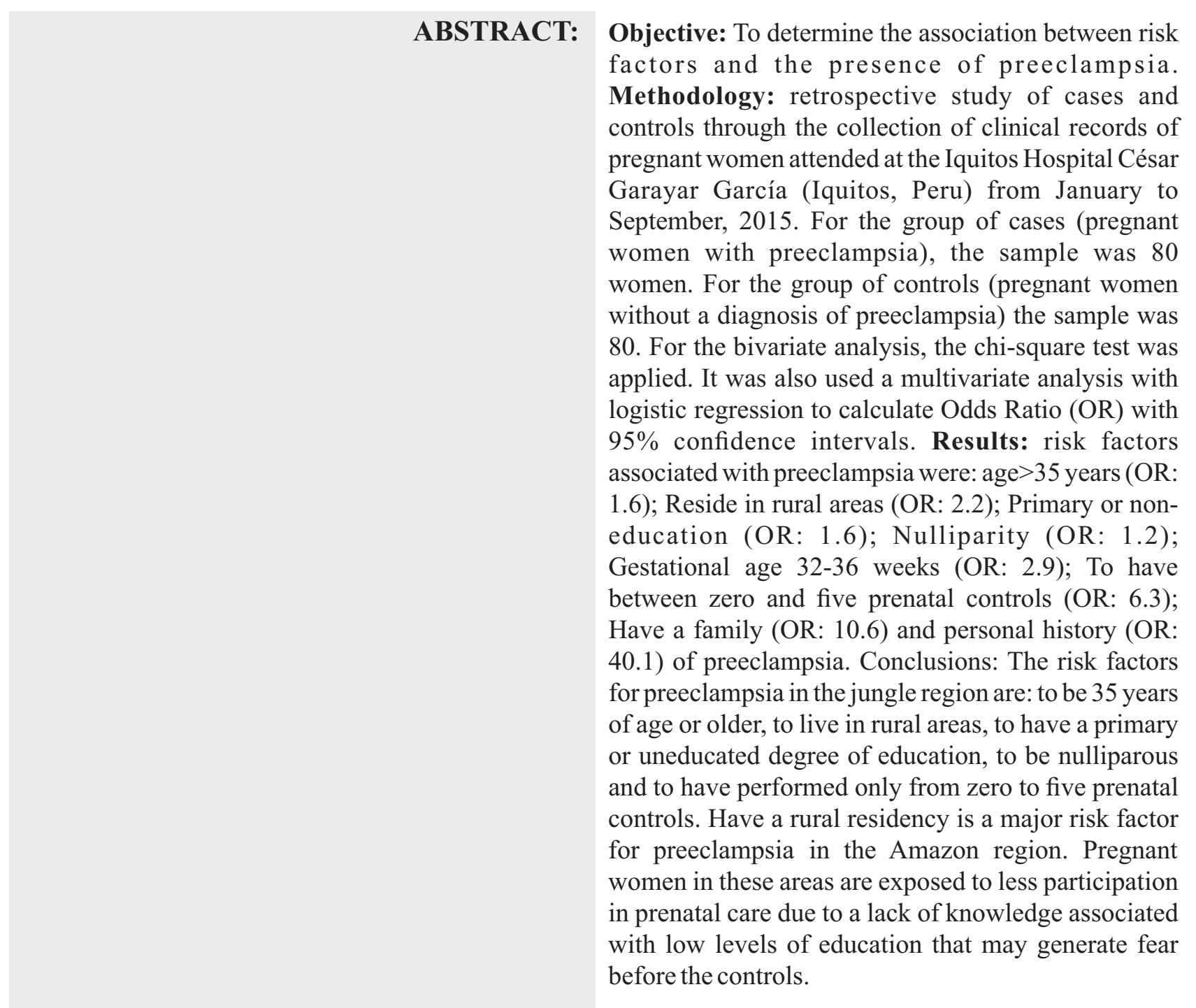

KEY WORDS: Preeclampsia; Risk Factors; Associated Factors. 